\title{
Prevalencia y conocimientos de estudiantes de enfermería de una universidad pública sobre el cuidado a la persona que consume drogas lícitas ${ }^{1}$
}

Josue Arturo Medina Fernández ${ }^{2}$

Laura Dioné Ortiz Gómez ${ }^{3}$

\section{Institución: Universidad Autónoma de Yucatán}

\section{RESUMEN}

Introducción. El abuso de drogas lícitas se constituye en uno de los principales problemas de salud pública a nivel internacional y nacional. En México, la Encuesta Nacional de Adicciones 2011 reporta que el 53,9\% de adultos consume alcohol y el 19,9\%, tabaco; en Yucatán, la proporción de adultos que consumía alcohol fue de $52,6 \%$ y tabaco, de 17,7\%. El objetivo del estudio fue determinar la prevalencia y el nivel de conocimientos de estudiantes de enfermería de una universidad pública sobre el cuidado a la persona que consume drogas lícitas.

Método. Utilizando una metodología cuantitativa, de corte transversal; con muestreo aleatorio estratificado por semestre, y selección por muestreo aleatorio simple. Se aplicó el cuestionario CDCCP, para obtener datos sociodemográficos, así como la prevalencia en alcohol y tabaco. Se aplicó el instrumento NEADA $\alpha=0.70$ para medir el nivel de conocimientos respecto del cuidado a la persona que consume drogas lícitas. Se encuestó a 74 estudiantes, $56.8 \%$ mujeres y un $43.2 \%$ hombres; $x=21.14$, $\pm=2.462$ años.

Resultados. La prevalencia del consumo de los estudiantes fue de $81,1 \%$ de alcohol y 51,1\%, de tabaco. De acuerdo con el NEADA, se obtuvo un $\mathrm{x}=20,15$ puntos $\mathrm{y} \pm=2,280$ puntos, que corresponde a un nivel medio de conocimientos.

Conclusión. Los estudiantes de pregrado presentaron una prevalencia global elevada del consumo y un nivel de conocimientos medio sobre el cuidado al paciente que consume drogas licitas, lo que sugiere, el desarrollo de programas de salud dirigidos a los estudiantes a fin de reducir o evitar el aumento de la prevalencia del consumo. Por otro lado, se observa la necesidad de reforzar los conocimientos del estudiante de pregrado para la atención a pacientes consumidores, las condiciones crónicas, psicológicas y sociales inherentes.

Palabras clave: adquisión-de-conocimientos, estudiantes-de-enfermería, uso-de-drogas, prevalencia

\footnotetext{
${ }^{1}$ Fecha de recepción: 17 de mayo del 2016

Fecha de aceptación: 15 de noviembre del 2016

${ }^{2}$ Serviço Social estagiário Bacharelado em Enfermagem. Escola de Enfermagem.Correo electrónico: enfermería Ccba@hotmail.com

${ }^{3}$ Professor, Pesquisador. Mestre em Investigação em Saúde. Faculdade de Enfermagem da Universidade de Yucatan. Correo electrónico: laura.ortiz@,correo.uady.mx
} 


\title{
Prevalence and knowledge of nursing students of a public university on the care of the person who consumes licit drugs 1
}

Institution: Autonomous University of Yucatan

\author{
Josue Arturo Medina Fernández ${ }^{2}$ \\ Laura Dioné Ortiz Gómez ${ }^{3}$
}

\begin{abstract}
Introduction. Licit drug abuse is one of the major public health problems at the international and national levels. In Mexico, the 2011 National Addiction Survey reports that 53,9\% of adults consume alcohol and 19,9\%, tobacco; in Yucatan, the proportion of adults who consumed alcohol was 52,6\% and tobacco, 17,7\%. The aim of the study was to determine the prevalence and level of knowledge of nursing students of a public university on care for the person who consumes licit drugs.
\end{abstract}

Method. Using a quantitative, cross-sectional methodology; with stratified random sampling by semester, and selection by simple random sampling. The CDCCP questionnaire was used to obtain sociodemographic data, as well as the prevalence in alcohol and tobacco. The instrument NEADA $\alpha=0.70$ was used to measure the level of knowledge regarding the care of the person who consumes licit drugs. We surveyed 74 students, 56,8\% female and $43,2 \%$ male; $x=21.14, \pm=2.462$ years.

Results. The prevalence of student consumption was $81,1 \%$ of alcohol and $51,1 \%$ of tobacco. According to the NEADA, we obtained $\mathrm{x}=20.15$ points and $\pm=2.280$ points, corresponding to an average level of knowledge.

Conclusion. The students had a high overall prevalence of consumption and an average level of knowledge about patient care that consumes legal drugs, suggesting the development of student-directed health programs in order to reduce or avoid the increase of the prevalence of consumption.

Key words: Acquisition-of-knowledge, drug-use, student-nursing, prevalence

\footnotetext{
${ }^{1}$ Date of receipt: May 17, 2016

Date of acceptance: November 15, 2016

${ }^{2}$ Intern of Social Service of Nursing Degree. Faculty of Nursing. Email: enfermería Ccba@hotmail.com

${ }^{3}$ Research professor. Master in Research for Health. Faculty of Nursing, Autonomous University of Yucatan. Email:

laura.ortiz@,correo.uady.mx
} 


\title{
Prevalência e conhecimento de estudantes de enfermagem de uma universidade pública no cuidado à pessoa consumir drogas legais ${ }^{1}$
}

\author{
Josue Arturo Medina Fernández ${ }^{2}$ \\ Laura Dioné Ortiz Gómez ${ }^{3}$
}

Instituição: Universidade Yucatan

\section{RESUMO}

Introdução. Abuso de drogas lícitas constitui um dos principais problemas de saúde pública a nível nacional e internacional. No México, a Pesquisa Nacional de Vícios 2011 informou que 53,9\% dos adultos consomem álcool e 19,9\%, rapé; em Yucatan, a proporção de adultos que consumiram álcool foi de 52,6\% e rapé, 17,7\%. O objetivo do estudo foi determinar a prevalência e nível de conhecimento dos estudantes de enfermagem de uma universidade pública no cuidado à pessoa consumir drogas legais.

Método. Usando uma metodologia quantitativa, transversal; com a amostragem aleatória estratificada por semestre, e seleção por amostragem aleatória simples. o questionário CDCCP foi aplicado para obter dados sóciodemográficos, bem como a prevalência de álcool e rapé. o instrumento NEADA $\alpha=0,70$ foi aplicado para medir o nível de conhecimento sobre o cuidado à pessoa consumir drogas legais. Foram pesquisados 74 alunos, 56,8\% de mulheres e $43,2 \%$ homens; $x=21,14, \pm=2462$ anos.

Resultados. A prevalência de alunos foi de $81,1 \%$ e $51,1 \%$ de álcool, de tabaco. De acordo com um NEADA x $=$ 20,15 pontos foi obtido $\mathrm{e} \pm=2.280$ pontos, o que corresponde a um nível médio de conhecimentos.

Conclusão. estudantes universitários mostrou uma alta prevalência de uso e um nível médio de conhecimento sobre cuidados de consumir drogas legais dos pacientes, sugerindo o desenvolvimento de programas de saúde para os alunos, a fim de reduzir ou evitar o aumento a prevalência. Por outro lado, a necessidade de reforçar o conhecimento dos consumidores de graduação cuidar de pacientes, condições crônicas, psicológicos e sociais inerentes observado.

Palavras-chave: prevalência, conhecimentos, estudantes de enfermagem, cuidado, drogas lícitas.

\footnotetext{
${ }^{1}$ Data de recepção: 17 de maio de 2016

Data de aceitação: 15 de novembro de 2016

${ }^{2}$ Intern of Social Service of Nursing Degree. Faculty of Nursing. Email: enfermería Ccba@hotmail.com

${ }^{3}$ Research professor. Master in Research for Health. Faculty of Nursing, Autonomous University of Yucatan. Email:

laura.ortiz@,correo.uady.mx
} 


\section{INTRODUCCIÓN}

El consumo de drogas es un problema de salud pública a nivel mundial que ha aumentado en los últimos veinte años, es una de las causas principales que perturba la vida en comunidades, altera la convivencia en las familias y la sociedad, además de arriesgar y cuestionar la importancia de los valores como guías del comportamiento y convivencia social armónica ${ }^{1}$.

El consumo nocivo de alcohol genera una carga sanitaria, social y económica considerable para el conjunto de la sociedad. En el 2012, se atribuyó 3.3 millones de defunciones en el mundo al consumo de alcohol ${ }^{1}$. En México, en la Encuesta Nacional de Adicciones, $2011^{2}$ se señaló que el consumo de alcohol alguna vez en la vida en la edad de entre 12 a 65 años fue de 42,9\% ${ }^{2}$. En Yucatán la proporción de adultos que consumía alcohol fue de $52,6 \%, 61,5 \%$ de hombres y $44,3 \%$ de mujeres ${ }^{3}$.

Comparado con el consumo de tabaco, en el mundo cerca de 5 millones son consumidores directos y más de 600000 son no fumadores expuestos al humo ajeno ${ }^{4}$, en cambio, en México el 15,9\% de la población que fuma es mayor de 15 años, porcentaje que representa 10.9 millones de mexicanos ${ }^{5}$. En Yucatán, se estimó que el 26,7\% de la población mayor de 20 años de edad ha fumado más de 100 cigarrillos en toda su vida, con un porcentaje mayor en hombres $(46,3 \%)$ que en mujeres $(8,5 \%)^{3}$.

La evidencia muestra que el consumo de drogas lícitas en estudiantes de enfermería es alto, tal como se expone en un estudio realizado en México, cuya muestra corresponde a edades entre los 16 y 40 años y una prevalencia de consumo de $76,1 \%$ en alcohol y $37,7 \%$, en tabaco. Por otra parte, en Colombia la muestra tuvo un rango de edad de 17 a 21 años, la prevalencia fue del $88 \%$ en alcohol y $24 \%$ tabaco ${ }^{6,7}$.

El conocimiento se refiere a contenidos del cuidado de enfermería para la atención de personas con dependencia a drogas, del cual se deriva la evidencia científica que sustenta la práctica profesional. Uno de los 
factores que pueden modificar los conocimientos del cuidado son las creencias, las cuales son ideas de sentido común que incluyen pensamientos sociales, valores, relaciones y estereotipos que orientan la acción de los individuos. ${ }^{8}$. Estudios previos han mostrado que el conocimiento en las adicciones en los estudiantes de enfermería es todavía limitado y que la comprensión sobre las razones de los pacientes respecto del uso y la dependencia es incompleta ${ }^{9}$.

El profesional de enfermería tiene dificultades en el cuidado de las personas que abusan de las drogas, lo cual lo limita al brindar el cuidado enfermero efectivo, y eficaz y persiste una actitud negativa en cuanto a las personas que presentan problemas con el abuso ${ }^{10}$.

Durante su proceso académico o laboral, los estudiantes de enfermería están inmersos en un contexto cultural tanto protector como de riesgo en cuanto al consumo de drogas, dado que enfrentan diversas situaciones físicas, emocionales y sociales que pueden aumentar la probabilidad de consumo con el fin de mitigar tales situaciones ${ }^{11}$.

Pese a la existencia de programas para prevenir las adicciones o el consumo abusivo de alcohol y tabaco, el descenso en las estadísticas de consumo es leve, lo cual afecta de manera directa el costo de los insumos y la calidad de atención a los pacientes ingresados ${ }^{12}$, hecho que resalta la poca información relacionada con el desarrollo de competencias para el cuidado de estos pacientes por parte del personal de enfermería.

La presente investigación tiene como objetivo identificar la prevalencia y el nivel de conocimientos sobre el consumo de drogas lícitas en los estudiantes de enfermería de una universidad pública, a fin de determinar las áreas de oportunidad en cuanto a la formación de este recurso humano, mediante la transferencia de los conocimientos y el fortalecimiento las habilidades y capacidades que se requiere incluir y aprender en el proceso de formación profesional, dado que contribuyen a su vez en la calidad del cuidado de enfermería. Los resultados del estudio podrán generar intervenciones aplicadas a la prevención de drogas lícitas y sugerir la implementación de asignaturas clave que permitan la formación integral y profesional de los estudiantes de enfermería en el área de adicciones.

\section{MATERIALES Y MÉTODO}

La población de estudio fueron los estudiantes la Licenciatura en Enfermería de la Universidad Autónoma de Yucatán (UADY), formada por 334 alumnos, de los cuales, mediante un muestreo aleatorio estratificado por semestre, se seleccionó una muestra de 74 estudiantes, considerando ocho estratos que corresponden a los ocho semestres de la carrera.

Este tipo de muestreo está representado por la formula $\mathrm{n}=\frac{\sum \mathrm{Nn} P \mathrm{Qn}}{\mathrm{N}_{\mathrm{K} 2} 2+\frac{1}{\mathrm{~N}} \sum \mathrm{Nn} P n \mathrm{Qn}}$ utilizando un nivel de confianza del 95\%, una potencia de 0.90 , un coeficiente de determinación del 5\%. La selección de la muestra fue por muestreo aleatorio simple estratificado, integrada de la siguiente manera: diez estudiantes de $1^{\text {er }}$ semestre, diez de $2^{\circ}$ semestre, nueve de $3^{\text {er }}$ semestre, ocho de $4^{\circ}$ semestre, doce de $5^{\circ}$ semestre, nueve de $6^{\circ}$ semestre, ocho de $7^{\circ}$ semestre y ocho de $8^{\circ}$ semestre. 
La muestra fue elegida por los siguientes criterios de selección: ser alumno regular de la licenciatura, estar inscrito al curso enero- diciembre 2014. Exclusión: ser menor de edad, no haber aceptado participar voluntariamente en el estudio. Eliminación: haber contestado respuestas ambiguas o confusas, retirarse del estudio previamente a la aplicación del instrumento.

Posterior, se solicitó a los estudiantes su participación, no sin antes explicarles los objetivos de estudio y solicitarles la firma del consentimiento informado. Los datos estadísticos se obtuvo mediante la cédula de datos personales y de consumo de alcohol (CDCCP), el cual mide los datos sociodemográficos, así como también el consumo de alcohol y otras drogas; también se utilizó el instrumento Nursing Education in Alcohol and Drug Education (NEADA) $(\alpha=0.70)$ traducido al español por intérpretes reconocidos del Ministerio de Educación de Colombia. Dicho instrumento se divide en dos partes: la primera es de conocimientos y la segunda, de creencias, por lo tanto, en esta investigación solo se pretende utilizar la primera parte con la variable conocimiento que consta de 14 preguntas y la siguiente escala: De acuerdo (2pts), Indiferente (1 pto) y Desacuerdo (0 pts.).

Para el procesamiento de la información se empleó el programa Stadistic Package for the Social Science (SPSS) de Windows versión 20, del que se obtuvo estadísticas descriptivas, así como frecuencias, porcentajes y medidas de tendencia central (media y desviación estándar).

La puntuación de los conocimientos según el NEADA tiene un intervalo de 28-0, por lo tanto, los estudiantes que obtuvieron una puntuación de 28-23 cuentan con un conocimiento alto; de 22-16, es medio y de 15-0, bajo.

\section{Consideraciones éticas}

El presente proyecto, se apegó al reglamento de la Ley General de Salud de los Estados Unidos Mexicanos en materia de investigación en seres humanos, descritos en el título quinto, capitulo único artículo 100 apartado 4 y 6, así como a la Norma oficial mexicana NOM-012-SSA3-2012. Se convocó a los estudiantes a una reunión informativa sobre el estudio y, quienes aceptaron participar firmaron el consentimiento informado, mismo que fue firmado por dos testigos.

\section{RESULTADOS}

Se aplicó el instrumento de recolección de datos a un total de 74 estudiantes de la Licenciatura en Enfermería de la Universidad Autónoma de Yucatán que cumplieron con los criterios de inclusión. A partir del instrumento CDCCP, se obtuvo que el porcentaje de mujeres que participaron fue de un 56,8\% (42), frente al $43,2 \%$ de los hombres; respecto del estado civil, en su mayoría eran solteros $(93,2 \%)$. La edad de los participantes oscila entre los 18 y los 29 años, con un $x=21.14, \pm=2.462$ años.

Para los estudiantes de la licenciatura, la prevalencia del consumo de las drogas lícitas fue de un $81,1 \%$ (60) respecto del alcohol y de 54,1\% (40), en cuanto a tabaco (véase tabla 1 ). 


\section{Revista Electrónica Enfermeria Actual en costa Rica}

Tabla 1. Universidad Autónoma de Yucatán. Distribución de la prevalencia de alcohol y tabaco en estudiantes de enfermería. (Frecuencias absolutas $=f i$, relativas $=F I$ )

\begin{tabular}{|c|c|c|c|c|c|c|c|}
\hline & \multicolumn{4}{|c|}{ Sexo } & \multirow{2}{*}{\multicolumn{2}{|c|}{ Total }} \\
\hline & & \multicolumn{2}{|c|}{ Hombre } & \multicolumn{2}{|c|}{ Mujer } & & \\
\hline & & $f i$ & $F i$ & $f i$ & $F i$ & $f i$ & $F i$ \\
\hline \multirow[t]{2}{*}{ Prevalencia del consumo de alcohol } & Sí consume & 26 & 35,1 & 34 & 45,9 & 60 & 81,1 \\
\hline & No consume & 6 & 8,1 & 8 & 10,8 & 14 & 18,9 \\
\hline \multirow[t]{2}{*}{ Prevalencia del consumo de tabaco } & Sí consume & 19 & 25,7 & 21 & 28,4 & 40 & 54,1 \\
\hline & No consume & 13 & 17,6 & 21 & 28,4 & 34 & 45,9 \\
\hline
\end{tabular}

Fuente: $\mathrm{CDCCP}, \mathrm{n}=74$

La relación de edad en la cual los estudiantes consumieron por primera vez tabaco estuvo entre los 10años con un $1,4 \%(f i=1)$ y $\operatorname{los} 20$ años $8,1 \%(f i=6)$, con una $\mathrm{x}=16.73$ años y una $\pm=2.522$, mientras que la relación de edad e inicio de consumo de alcohol fue entre los 12 años $4,1 \%(f i=3)$ y los 23 años $1,4 \%(f i=1)$, con una $\mathrm{x}=16.45$ años y una $\pm=2.078$.

Al establecer una relación con la escala de formación de enfermería en conocimientos de alcohol y tabaco (NEADA), sus conocimientos tuvieron una $\mathrm{x}=20.15$ pts. $\mathrm{y} \pm=2.280$, con una puntuación mínima 16 pts. $2,7 \%$ $(f i=2)$, y una máxima de 26 pts. $1,4 \%(f i=1)$, es decir, de acuerdo con este instrumento, los estudiantes tienen un nivel medio de conocimientos.

Tales puntuaciones difieren entre hombres y mujeres: los primeros obtienen una media $\mathrm{x}=19.65$ puntos, mientras que la de las mujeres fue de $\mathrm{x}=17.23$, como se aprecia en la tabla 2. Lo anterior significa que los hombres cuentan con un mayor nivel de conocimientos respecto de las mujeres; sin embargo, ambas puntuaciones se ubican en un nivel de conocimientos medio, de acuerdo con el instrumento.

Tabla 2. Universidad Autónoma de Yucatán. Distribución de las puntuaciones generales de NEADA según el sexo. $($ Frecuencias absolutas $=f i$, relativas $=F I$ )

\begin{tabular}{c|c|c|c|c|c|c}
\hline \multirow{2}{*}{$\begin{array}{c}\text { Puntuación } \\
\text { NEADA }\end{array}$} & \multicolumn{4}{c|}{ Sexo } & \multicolumn{2}{c}{} \\
\cline { 2 - 8 } \multicolumn{2}{c|}{} & \multicolumn{2}{|c|}{ Hombres } & \multicolumn{2}{c}{ Mujeres } & \multicolumn{2}{c}{ Total } \\
\cline { 2 - 8 } & $\boldsymbol{f i}$ & $\boldsymbol{F i}$ & $\boldsymbol{f i}$ & $\boldsymbol{F i}$ & $\boldsymbol{f i}$ & $\boldsymbol{F i}$ \\
\hline 16 & 1 & 1,4 & 1 & 1,4 & 2 & 2,7 \\
\hline 17 & 3 & 4,1 & 3 & 4,1 & 6 & 8,1 \\
\hline 18 & 8 & 10,8 & 5 & 6,8 & 13 & 17,6 \\
\hline 19 & 2 & 2,7 & 6 & 8,1 & 8 & 10,8 \\
\hline 20 & 10 & 13,5 & 7 & 9,5 & 17 & 23 \\
\hline 21 & 1 & 1,4 & 7 & 9,5 & 8 & 10,8 \\
\hline 22 & 4 & 5,4 & 3 & 4,1 & 7 & 9,5 \\
\hline 23 & 2 & 2,7 & 6 & 8,1 & 8 & 10,8 \\
\hline 24 & 0 & 0 & 1 & 1,4 & 1 & 1,4 \\
\hline
\end{tabular}




\begin{tabular}{c|c|c|c|c|c|c|c}
\hline 25 & 1 & 1,4 & 2 & 2,7 & 3 & 4,1 \\
\cline { 2 - 6 } & 26 & 0 & 0 & 1 & 1,4 & 1 & 1,4 \\
\hline \multirow{2}{*}{ Total } & 32 & 43,2 & 42 & 56,8 & \multirow{2}{*}{$100 \%$} \\
\cline { 2 - 4 } & \multicolumn{2}{|l|}{$\mathrm{x}=19.65$ pts. } & \multicolumn{2}{|c|}{$\mathrm{x}=17.23$ pts. } & \\
\hline
\end{tabular}

Fuente: NEADA, $n=74$

Con respecto al nivel de conocimientos, el 100\% de los estratos se ubica en un nivel medio; no obstante, se observó que los estudiantes de séptimo semestre presentan una leve ventaja en las puntuaciones sobre el nivel de conocimientos, la cual radica en haber cursado la asignatura de cuidado enfermero en las adicciones como parte de las asignaturas optativas (Véase tabla 3 ).

Tabla 3. Universidad Autónoma de Yucatán. Distribución de la puntuación NEADA por semestre de la Licenciatura en Enfermería.

\begin{tabular}{c|c|c|c|c}
\hline Semestre/NEADA & $\mathbf{X}$ & $\mathbf{S}$ & $\begin{array}{c}\text { Valor } \\
\text { mínimo (pts) }\end{array}$ & $\begin{array}{c}\text { Valor } \\
\text { Máximo (pts) }\end{array}$ \\
\hline $1^{\circ}$ & 19.80 & 2.251 & 17 & 24 \\
\hline $2^{\circ}$ & 19.30 & 2.111 & 16 & 23 \\
\hline $3^{\circ}$ & 19.22 & 1.986 & 16 & 23 \\
\hline $4^{\circ}$ & 20.50 & 2.619 & 17 & 25 \\
\hline $5^{\circ}$ & 20.67 & 2.348 & 18 & 25 \\
\hline $6^{\circ}$ & 19.89 & 1.691 & 17 & 22 \\
\hline $7^{\circ}$ & 21.50 & 3.162 & 17 & 26 \\
\hline $8^{\circ}$ & 20.50 & 1.852 & 18 & 23 \\
\hline
\end{tabular}

Fuente: NEADA, $n=74$

Por otra parte, del total de los participantes, 13 obtuvieron un nivel alto de conocimientos, 59, uno medio y 2, uno bajo. Tomando en cuenta a los que sí consumen alcohol, la media del nivel de conocimientos según la NEADA fue de $x=20.08$ pts., mientras que el nivel de conocimientos para los que no consumen fue de $x=20.51$ pts., en la que los hombres obtuvieron mayor conocimiento, lo cual muestra que lo estudiantes que no consumen alcohol y tabaco tiene un mayor nivel de conocimientos con respecto a los que sí consumen.

\section{DISCUSIÓN}

La prevalencia del consumo de alcohol y tabaco en este estudio fue mayor en un $50 \%$ y el nivel de conocimientos en relación con el cuidado a la persona que consume drogas lícitas corresponde a nivel medio. 
Dichos resultados indican que el consumo en ambas drogas lícitas hace de los estudiantes una población vulnerable para su consumo. En algunas ocasiones, la universidad se convierte en un punto para iniciar el consumo de drogas adictivas, debido a las "cualidades" que les atribuyen en cuanto al aumento de la creatividad, el desarrollo intelectual o a facilitar la socialización ${ }^{13}$.

El contexto del estudio es similar a otros, en los que la mayoría de los participantes son mujeres (56,8\%), tal como sucede en escuelas de varios países, como en Perú en las que el $100 \%$ de las entrevistadas eran mujeres, mientras que en Colombia fue del $81,1 \%$ y en Monterrey, México, de un $68,7 \%^{11,14,15}$. Lo anterior se puede atribuir a que históricamente la profesión fue iniciada por mujeres y actualmente el entorno sociocultural asigna atributos exclusivos para el sexo femenino.

Con respecto al consumo de alcohol, el $81,1 \%$ de la muestra lo ha consumido y ese porcentaje, en su mayoría, está constituido por mujeres. La alta prevalencia se puede observar en el estudio realizado en Lima Perú en el que se estudió a los alumnos del segundo año y obtuvieron un porcentaje mayor del $50 \%$, mientras que en Río de Janeiro, Brasil, en una universidad privada fue del 67,7\% y en Veracruz, México, fue de 66,7\% ${ }^{15,16,17}$. Los estudiantes universitarios consumen alcohol por carencia de información correcta, estrés y falta de actividades recreativas, puesto que presentan una dificultad para relajarse y por ello recaen en el alcohol ${ }^{16}$.

De los participantes de este estudio, el 54,1\% consumió alguna vez tabaco, de lo que se colige que el consumo es mayor en los estudiantes de dos universidades privadas de Monterrey, México (56\%) aunque fue menor en un estudio realizado en la Universidad de Antioquia $(39,5 \%)^{11,14}$. Lo anterior podría radicar en que la Facultad de Enfermería de la UADY tiene el programa Edificio 100\% libre humo de tabaco, lo cual podría considerarse un efecto preventivo menor en los estudiantes universitarios ${ }^{18}$.

Los conocimientos obtenidos por la mayoría de los encuestados corresponde a nivel medio, debido a que el plan de estudios cuenta con el curso optativo de cuidado enfermero en las adicciones y en la asignaturas de salud mental existen temas relacionados, aunque falta la iniciativa e interés por parte de los estudiantes respecto de escoger las asignatura mencionadas para mejorar los conocimientos del cuidado a las personas que consumen drogas lícitas que actualmente han incrementando, sobre todo en el Estado de Yucatán, de acuerdo a las estadísticas de la Encuesta Nacional de Salud y Nutrición $2012^{3}$.

En torno al tema, dos estudios presentaron una similitud de conocimientos medio: uno fue realizado en Querétaro y evaluó conocimientos conceptuales, procedimentales y actitudinales del problema del fenómeno de las adicciones: el segundo se desarrolló en Santa Martha, Colombia, y se enfocó en sustancias psicoactivas ${ }^{19,20}$. En este último, las evidencias relacionadas con el nivel de conocimientos, demuestran que estos puntajes están por debajo de la media establecida por el propio instrumento de evaluación, lo cual sugiere que no asocian o no perciben la importancia de estudiar esta temática, por ende, el plan curricular debe incrementar el enfoque al cuidado de las adicciones, con el fin de capacitar a los estudiantes de pregrado y permitir detección temprana, intervención preventiva para ellos y la población.

Con respecto a la prevalencia en el consumo de alcohol y tabaco de los estudiantes entrevistados, fue elevado, lo cual es alarmante, ya que los estudiantes pueden desarrollar factores de riesgo para padecer 
alcoholismo y tabaquismo, por lo que se sugiere reforzar los conocimientos enfocados en el desarrollo de competencias de promoción y educación para la salud en las adicciones.

La educación superior debe brindar respuestas a la complejidad de los problemas de la sociedad que generan transformaciones en las estructuras sociales y que exigen cambios en la educación, entonces, es evidente la necesidad de un aprendizaje distinto e innovador que además permita al futuro egresado enfrentar los nuevos retos de la oferta y demanda del mercado laboral de las profesiones con competitividad y calidad ${ }^{21}$. Desde tal perspectiva, una de las competencias por desarrollar desde el primer semestre de la Licenciatura en Enfermería es la atención en el primer nivel de atención, con las funciones de promotor y educador para la salud; sin embargo, las evidencias de este estudio sugieren que los estudiantes no se han identificado con las funciones y roles del profesional del área de la salud. Lo anterior hace referencia a que la formación del profesional de enfermería es limitada en el tema de las adicciones y requiere de una participación como parte del equipo interdisciplinario que fundamenta la modificación curricular y revisión del programa vigente ${ }^{10}$.

Los resultados evidencian la necesidad de implementarse programas o estrategias de promoción de la salud y prevención del consumo de sustancias psicoactivas, con el propósito de evitar que la prevalencia del consumo aumente, afecte el proyecto de vida de los estudiantes y ocasione problemas escolares, familiares, sociales, económicos y morales 22 .

Por otra parte, es necesario desarrollar investigaciones que analicen la calidad de los cuidados en salud de parte de las enfermeras a los drogodependientes en nuestro entorno sanitario y que profundicen en el impacto de estas actitudes en la atención proporcionada a los drogodependientes ${ }^{23}$.

\section{CONCLUSIÓN}

Los estudiantes de pregrado presentaron una prevalencia global elevada del consumo y un nivel de conocimientos medio sobre el cuidado al paciente que consume drogas licitas, lo que sugiere, el desarrollo de programas de salud dirigidos a los estudiantes a fin de reducir o evitar el aumento de la prevalencia del consumo. Por otro lado, se observa la necesidad de reforzar los conocimientos del estudiante de pregrado para la atención a pacientes consumidores, las condiciones crónicas, psicológicas y sociales inherentes.

Es necesario el desarrollo de investigaciones que aborden la temática del consumo desde otros enfoques metodológicos a fin de analizar la calidad de los cuidados en salud proporcionados por enfermería a los consumidores de drogas licitas y que profundicen en el impacto de las actitudes en la atención.

\section{Conflicto de interés}

Los autores declaran que no existe conflicto de interés en el desarrollo y publicación de este estudio. 


\section{AGRADECIMIENTOS}

A la Facultad de Enfermería de la Universidad Autónoma de Yucatán por el apoyo y la accesibilidad en el desarrollo de dicha investigación.

\section{REFERENCIAS BIBLIOGRÁFICAS}

1. Organización Mundial de la Salud [Internet]. (2015a). Alcohol. [Acceso el 23 de junio de 2014]. Disponible en http://www.who.int/mediacentre/factsheets/fs349/es/

2. Encuesta Nacional de Adicciones 2011. [Internet]. México D. F.: Instituto Nacional de Psiquiatría Ramón de la Fuente Muñiz. [Acceso el 5 de junio del 2014]. Disponible en http://www.conadic.salud.gob.mx/pdfs/ENA_2011_DROGAS_ILÍCITAS_pdf

3. Encuesta Nacional de Salud y Nutrición 2012. [Internet]. México D. F.: Instituto Nacional de Salud Pública. [Acceso el 23 de junio de 2014] Disponible en http://ensanut.insp.mx

4. Organización Mundial de la Salud. [Internet]. (2015b). Tabaquismo. [Acceso el día 23 de junio del 2014]. Disponible en http://www.who.int/mediacentre/factsheets/fs339/es/

5. Comisión Federal de Mejora Regulatoria. [Internet]. Tabaquismo en México: análisis y recomendaciones de mejora regulatoria; 2012 Abril. Documento de Investigación en Regulación No. 2012-01. Disponible en http://www.cofemer.gob.mx/Varios/Adjuntos/14.05.2012/IAPA_COFEMER_TABAQUISMO_EN_MEX ICO 2012.pdf

6. López-Maldonado MC, Villar MC, Da Silva EC. Consumo de drogas lícitas en estudiantes de enfermería de universidad privada en Bogotá, Colombia. Rev. Latino-Am Enfermagem [Internet]. 2011 May/Junio [Acceso el 23 de junio del 2014] 19:707-713. DOI: 10.1590/S0104-11692011000700007. Disponible en http://www.scielo.br/scielo.php?script=sci arttext\&pid=S0104-11692011000700007

7. Martínez MR., Coronado MJ, Betancourt MC, Díaz A, Gallegos J. Drogas lícitas e ilícitas: consumo de los estudiantes en una Facultad de Enfermería. Enf Neurol (Mex) [Internet]. 2011 [Acceso 14 de junio del 2014]; 10(3):130-134. Disponible en http://www.medigraphic.com/pdfs/enfneu/ene-2011/ene113d.pdf

8. Moscovisi S. Psicología Social II pensamiento y vida social psicología social y problemas sociales. Cognición y desarrollo humano. Paris: Ed. Paidós; 1984. 372 p.

9. Vargas M, Arena C, Da Silva E. Conocimientos de estudiantes de enfermería sobre alcohol y drogas. Rev. Latino-Am. Enfermagem [Internet]. 2010 May/Jun [Acceso el 10 de junio del 2014]; 18 (spe):529-534. DOI: $10.1590 /$ S0104-11692010000700007. Disponible en http://www.scielo.br/scielo.php?script=sci arttext\&pid=S0104-11692010000700007 
10. García-Cardona M, y Ramírez-Elías A. La educación de enfermería y las adicciones. Una revisión de la literatura. Rev. Enferm IMSS [Internet]. 2010 [Acceso el 22 de junio del 2014]; 18(1):35-42. Disponible en http://new.medigraphic.com/cgi-bin/resumen.cgi?IDARTICULO=29737

11. Telumbre-Terrero JY, Esparza-Almanza SE, Alonso-Castillo BA, Alonso-Castillo MTJ. Consumo de alcohol y tabaco en estudiantes de enfermería. Rev Enfermería Actual en Costa Rica [Internet]. 2016 EneJun [Acceso el 22 de junio del 2014]; 30: 1-16. DOI: 10.15517/revenf.v0i30.22020. Disponible en http://www.revistas.ucr.ac.cr/index.php/enfermeria/article/view/22020/22498

12. Villatoro J, Gaytán F, Moreno M, Gutiérrez ML, Robles NO, Bretón M, et. al. Tendencias del uso de Drogas en la Ciudad de México: Encuesta de Estudiantes del 2009. Salud Ment [Internet]. 2011 mar/abr. [Acceso el 9 de junio del 2014] 34(2): 81-94. Disponible en http://www.scielo.org.mx/scielo.php?script=sci_arttext\&pid=S0185-33252011000200001

13. Quimbayo-Díaz JH, Olivella-Fernández MC. Consumo de marihuana en estudiantes de una universidad colombiana. Rev. de Salud Pública. [Internet]. 2013. [Acceso el 10 de junio de 2014];15(1): 32-43. Disponible en http://www.scopus.com/inward/record.url?eid=2-s2.0-84894588864\&partnerID=tZOtx3y1

14. Rodríguez MA, Pineda SA, Vélez LF. Características del consumo de tabaco en estudiantes de enfermería de la Universidad de Antioquia (Colombia). Invest. Educ. Enferm [Internet]. 2010 [Acceso el 14 de junio 2014]; 28(3): 370-383. Disponible en http://www.scielo.org.co/scielo.php?pid=S012053072010000300008\&script $=$ sci arttext\&tlng $=$ es

15. Tam E, Benedita C. El consumo de alcohol y el estrés entre estudiantes del segundo año de enfermería. Rev. Latino-Am Enfermagem [Internet]. 2010 may/jun [Acceso el 22 de junio del 2014] 18: 496-503. DOI: 10.1590/S0104-11692010000700003. Disponible en http://www.scielo.br/scielo.php?script=sci arttext\&pid=S0104-11692010000700003

16. Otero O. Ansiedad y consumo de alcohol en estudiantes universitarios [Tesis de licenciatura en Enfermería]. Minatitlán:Universidad Veracruzana; 2012.

17. Tavares-Jomar R, Dos Santos-Silva E. (2013). Consumo de bebidas alcohólicas en estudiantes de Enfermería. Aquichan [Internet]. 2013 [Acceso el 10 de junio del 2014]; 13(2): 226-233. DOI: 10.5294/aqui.2013.13.2.8. Disponible en http://aquichan.unisabana.edu.co/index.php/aquichan/article/view/2887

18. Morales G, Del Valler C, Berlmar C, Orellana Y, Soto A, Ivanovic D. Prevalencia de consumo de drogas en estudiantes universitarios que cursan primer y cuarto año. Rev. Méd. Chile [Internet]. 2011 [Acceso el 24 de octubre del 2014]; 139(12): 1573-1580. DOI: 10.4067/S0034-98872011001200006. Disponible en http://www.scielo.cl/scielo.php?script=sci_arttext\&pid=S0034-98872011001200006 


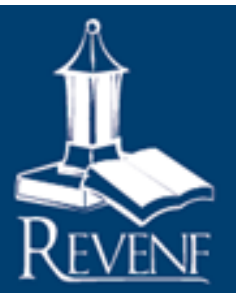

19. Camacho-Rodriguez D, Gonzalez-Ruiz G. (2015). Nursing knowledge on the use of psychoactive substances and their prevention. Revista Duazary [Internet]. 2015 Jul/Dic [16 de junio del 2014]; 12 (2): 93-99. Disponible en https://dialnet.unirioja.es/descarga/articulo/5156560.pdf

20. Garza B, Hernández MA, Gallegos R, Zamora A. Experiencias y conocimientos previos de los estudiantes de enfermería sobre los contenidos en la materia de adicciones. Ciencia@UAQ [Internet]. 2013 [Acceso el 10 de junio del 2014]; 6(2): 1-9. Disponible en http://www.uaq.mx/investigacion/revista_ciencia@uaq/ArchivosPDF/v6-n2/17Articulo.pdf

21. Alonso M, Armendáriz N, Guzmán F, López M, Villegas M, Álvarez A. Integración de contenidos temáticos de drogas al currículo de Doctorado en Ciencias de Enfermería: El caso de la Facultad de Enfermería de la Universidad Autónoma de Nuevo León. Edition. Monterrey: Universidad Autónoma de Nuevo León; 2014.

22. Páez AN, Solano SS, Durán M, Mancilla D, Suarez E, Melgarejo P, et al. Prevalencia del consumo de sustancias psicoactivas en estudiantes universitarios de la salud y ciencias sociales. CUIDARTE [Internet]. 2012 [Acceso el 9 de junio del 2014]; 3 (1): 334-341. DOI: 10.15649/cuidarte.v3i1.29. Disponible en http://www.revistacuidarte.org/index.php/cuidarte/article/view/29

23. Molina J, Hernández D, Sanz E, Clar F. Impacto de las actitudes de las enfermeras en la calidad de los cuidados en drogodependientes. Index de Enferm [Internet]. 2012 Oct/Dic [Acceso el 5 de agosto del 2014]; 21(4): 212-218. Disponible en http://scielo.isciii.es/scielo.php?script=sci_arttext\&pid=S113212962012003300008 